\title{
Power Ratio Gain Technique and General Integral Control
}

\author{
Baishun Liu \\ Academy of Naval Submarine, Qingdao, China \\ Email: baishunliu@163.com
}

Received 27 February 2015; accepted 10 April 2015; published 17 April 2015

Copyright (C) 2015 by author and Scientific Research Publishing Inc.

This work is licensed under the Creative Commons Attribution International License (CC BY). http://creativecommons.org/licenses/by/4.0/

(c) (i) Open Access

\begin{abstract}
In conjunction with general integral control, and synthesizing Singular perturbation and Equal ratio gain techniques, this paper proposes a new control design technique, named Power ratio gain technique, and then by Lyapunov method, theorem to ensure regionally as well as semi-globally asymptotic stability is established in terms of some bounded information. The highlight point is that it not only inherits all the essences of Singular perturbation and Equal ratio gain techniques but also makes up for their shortcomings, and then the conservatism of control input can be improved by compromising the Power ratio coefficients. Theoretical analysis, design example and simulation results show that Power ratio gain technique is a simple, practical and powerful tool to deal with the uncertain nonlinear system.
\end{abstract}

\section{Keywords}

General Integral Control, Nonlinear Control, Robust Control, Equal Ratio Gain Technique, Singular Perturbation Technique, Power Ratio Gain Technique, Output Regulation

\section{Introduction}

Faced with the complexity of uncertain nonlinear system, it is clear that we cannot expect a particular method to apply to all nonlinear systems [1]. Therefore, although there are Linearization technique, Gain scheduling technique, Singular perturbation technique, Feedback linearization technique, Sliding mode technique, Equal ratio gain technique and so on, nonlinear design tools, this paper still develops a new control design technique, named Power ratio gain technique, in conjunction with general integral control since integral control plays an irreplaceable role in the control domain because it ensures asymptotic tracking and disturbance rejection when exogenous signals are constants or planting parametric uncertainties appears.

For general integral control, there were various design techniques, such as Linearization technique [2], Sliding 
mode technique [3], Feedback linearization technique [4], Singular perturbation technique [5], Perturbation technique [6]-[9] and Equal ratio gain technique [10] [11]. Although Singular perturbation and Equal ratio gain techniques can all effectively deal with the uncertain nonlinear system, respectively, the results could be too conservative, especially for Singular perturbation technique. Therefore, it is of important significance to develop a new control design technique such that all the essences of Singular perturbation and Equal ratio gain techniques are preserved and their shortcomings are removed.

Motivated by the cognition above, in conjunction with general integral control, this paper proposes a new control design technique, named Power ratio gain technique. The main contributions are: 1 ) the perfect combination of Singular perturbation and Equal ratio gain techniques is achieved, and then the conservatism of control input can be improved by compromising the Power ratio coefficients; 2) by Lyapunov method, theorem to ensure regionally as well as semi-globally asymptotic stability is established in terms of some bounded information. Theoretical analysis, design example and simulation results show that Power ratio gain technique is a simple, practical and powerful tool to deal with the uncertain nonlinear system.

Throughout this paper, we use the notation $\lambda_{m}(A)$ and $\lambda_{M}(A)$ to indicate the smallest and largest eigenvalues, respectively, of a symmetric positive define bounded matrix $A(x)$, for any $x \in R^{n}$. The norm of vector $x$ is defined as $\|x\|=\sqrt{x^{\mathrm{T}} x}$, and that of matrix $A$ is defined as the corresponding induced norm $\|A\|=\sqrt{\lambda_{M}\left(A^{\mathrm{T}} A\right)}$.

The remainder of the paper is organized as follows: Section 2 describes the system under consideration, assumption, definition and propositions. Section 3 presents Power ratio gain technique and general integral control design. Example and simulation are provided in Section 4. Conclusions are presented in Section 5.

\section{Problem Formulation}

Consider the following controllable nonlinear system,

$$
\left\{\begin{array}{l}
\dot{x}_{1}=x_{2} \\
\dot{x}_{2}=x_{3} \\
\quad \vdots \\
\dot{x}_{n}=f(x, w)+g(x, w) u
\end{array}\right.
$$

where $x \in R^{n}$ is the state; $u \in R$ is the control input; $w \in R^{l}$ is a vector of unknown constant parameters and disturbances. The uncertain nonlinear functions $f(x, w)$ and $g(x, w)$ are all continuous in $(x, w)$ on the control domain $D_{x} \times D_{w} \subset R^{n} \times R^{l}$. We want to design a control law $u$ such that $x(t) \rightarrow 0$ as $t \rightarrow \infty$.

Assumption 1: There is a unique pair $\left(0, u_{0}\right)$ that satisfies the equation,

$$
0=f(0, w)+g(0, w) u_{0}
$$

so that $x=0$ is the desired equilibrium point and $u_{0}$ is the steady-state control that is needed to maintain equilibrium at $x=0$.

Assumption 2: Suppose that the functions $f(x, w)$ and $g(x, w)$ satisfy the following inequalities,

$$
\begin{gathered}
\|f(x, w)-f(0, w)\| \leq l_{f}^{x}\|x\| \\
0<g_{m}<g(x, w)<g_{M} \\
\|g(x, w)-g(0, w)\| \leq l_{g}^{x}\|x\| \\
\left\|f(0, w) g^{-1}(0, w)\right\| \leq \gamma_{g}^{f}
\end{gathered}
$$

for all $x \in D_{x}$ and $w \in D_{w}$, where $l_{f}^{x}, l_{g}^{x}, g_{m}, g_{M}$ and $\gamma_{g}^{f}$ are all positive constants.

For the purpose of this paper, it is convenient to introduce the following definition [12] and propositions [10].

Definition 1: $F_{\Phi}\left(a_{\Phi}, b_{\Phi}, x\right)$ with $a_{\Phi}>0, b_{\Phi}>0$, and $x \in R$ denotes the set of all continuous differential increasing function, $\Phi(x)$ such that 


$$
\begin{gathered}
\Phi(0)=0, \\
|\Phi(x)| \geq b_{\Phi} \quad \forall x \in R:|x|>a_{\Phi} \\
\mathrm{d} \Phi(x) / \mathrm{d} x>0 \quad \forall x \in R
\end{gathered}
$$

where $|\cdot|$ stands for the absolute value.

Figure 1 depicts the example curves for the functions belonging to the function set $F_{\Phi}$. For instance, for all $x \in R$, the functions, $\operatorname{arcsinh}(x), \tanh (x), a x+b x^{3}(a>0, b>0), \sinh (x), a x$ and so on, all belong to function set $F_{\Phi}$.

Proposition 1: a canonical system matrix can be designed to be Hurwitz as any row controller and its integrator gains increase with the same ratio.

Proposition 2: as any row controller and its integrator gains of a canonical system matrix tend to infinity with the same ratio, if it is always Hurwitz, and then the same row solutions of Lyapunov equation all tend to zero.

\section{Power Ratio Gain Technique and General Integral Control}

For developing Power ratio gain technique, general integral controller is given as,

$$
\left\{\begin{array}{l}
u=-\mu^{-1}\left(\varepsilon^{-n} \alpha_{1} x_{1}+\varepsilon^{-n+1} \alpha_{2} x_{2}+\cdots+\varepsilon^{-1} \alpha_{n} x_{n}+\varepsilon^{-1} \alpha_{n+1} \varphi(\sigma)\right)-\phi(x) \\
\dot{\sigma}=\left(\frac{\mathrm{d} \varphi(\sigma)}{\mathrm{d} \sigma}\right)^{-1} \mu^{-1}\left(\varepsilon^{-n} \beta_{1} x_{1}+\varepsilon^{-n+1} \beta_{2} x_{2}+\cdots+\varepsilon^{-2} \beta_{n-1} x_{n-1}+\varepsilon^{-1} \beta_{n} x_{n}\right)
\end{array}\right.
$$

where $\alpha_{i} \quad(i=1,2, \cdots, n+1), \quad \beta_{j} \quad(j=1,2, \cdots, n), \mu$ and $\varepsilon$ are all positive constants; $\varphi(\bullet)$ belongs to the function set $F_{\Phi} ; \phi(x)(\phi(0)=0)$ is used to attenuate the uncertain nonlinear action of $f(x, w)-f(0, w)$.

Assumptions 3: By the inequalities (3), (4) and definition of the controller (7), it is reasonable to suppose that the following inequality,

$$
\|f(x, w)-f(0, w)-g(x, w) \phi(x)\| \leq l_{f \phi}^{x}(x)
$$

holds for all $x \in D_{x}$ and $w \in D_{w}$. where $l_{f \phi}^{x}$ is a positive constant.

Thus, substituting (7) into (1), obtain the augmented system,

$$
\left\{\begin{array}{l}
\dot{x}_{1}=x_{2} \\
\dot{x}_{2}=x_{3} \\
\quad \vdots \\
\dot{x}_{n}=f(x, w)-g(x, w) \phi(x)-g(x, w) \mu^{-1}\left(\varepsilon^{-n} \alpha_{1} x_{1}+\varepsilon^{-n+1} \alpha_{2} x_{2}+\cdots+\varepsilon^{-1} \alpha_{n} x_{n}+\varepsilon^{-1} \alpha_{n+1} \varphi(\sigma)\right) \\
\dot{\varphi}(\sigma)=\mu^{-1}\left(\varepsilon^{-n} \beta_{1} x_{1}+\varepsilon^{-n+1} \beta_{2} x_{2}+\cdots+\varepsilon^{-2} \beta_{n-1} x_{n-1}+\varepsilon^{-1} \beta_{n} x_{n}\right)
\end{array}\right.
$$

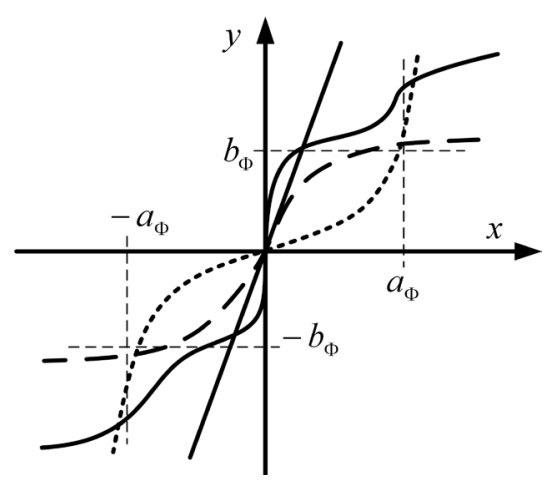

Figure 1. Example curves for the functions belonging to the function set $F_{\Phi}$. 
By Assumption 1 and choosing $\varepsilon^{-1} \mu^{-1} \alpha_{n+1}$ to be large enough, and then setting $\dot{x}=0$ and $x=0$ of the system (9), obtain,

$$
0=f(0, w)-g(0, w) \varepsilon^{-1} \mu^{-1} \alpha_{n+1} \varphi\left(\sigma_{0}\right)
$$

Therefore, we ensure that there is a unique solution $\sigma_{0}$, and then $\left(0, \sigma_{0}\right)$ is a unique equilibrium point of the closed-loop system (9) in the domain of interest. At the equilibrium point, $x=0$, irrespective of the value of $w$.

Now, defining

$$
\begin{gathered}
\eta=\left[\begin{array}{lllll}
\eta_{1} & \eta_{2} & \cdots & \eta_{n} & \eta_{n+1}
\end{array}\right]^{\mathrm{T}}, \\
\eta_{i}=\varepsilon^{-n+i} x_{i} \quad(i=1,2, \cdots, n), \\
\eta_{n+1}=\varphi(\sigma)-\varphi\left(\sigma_{0}\right),
\end{gathered}
$$

and substituting (10) into (9), obtain,

$$
\varepsilon \dot{\eta}=A \eta+\varepsilon F(x, w)
$$

where

$$
\begin{aligned}
& \eta=\left[\begin{array}{ll}
x^{\mathrm{T}} & \varphi(\sigma)-\varphi\left(\sigma_{0}\right)
\end{array}\right]^{\mathrm{T}} \\
& A=\left[\begin{array}{ccccc}
0 & 1 & \cdots & 0 & 0 \\
0 & 0 & \ddots & 0 & 0 \\
0 & 0 & \cdots & 1 & 0 \\
-\mu^{-1} \alpha_{1} & -\mu^{-1} \alpha_{2} & \cdots & -\mu^{-1} \alpha_{n} & -\mu^{-1} \alpha_{n+1} \\
\mu^{-1} \beta_{1} & \mu^{-1} \beta_{2} & \cdots & \mu^{-1} \beta_{n} & 0
\end{array}\right],
\end{aligned}
$$

and $F(x, w)$ is an $n+1 \times 1$ matrix, all its elements are equal to zero except for

$$
f_{n 1}=f(x, w)-f(0, w)-g(x, w) \phi(x)-[g(x, w)-g(0, w)] f(0, w) g^{-1}(0, w) .
$$

Moreover, it is worthy to note that the function $g(x, w)$ is integrated into $\alpha_{i}$ via a change of variable. This has not influence on the results if the inequality (4) holds and it can be taken as $g_{m}$ in the design. Therefore, it is omitted in all the following demonstrations.

By Proposition 1 proposed by [10], the system matrix $A$ can be designed to be Hurwitz for all $\alpha_{i}, \beta_{j}$ and $0<\mu<\mu^{*}$. Thus, by linear system theory, if the matrix $A$ is Hurwitz, and then for any given positive define symmetric matrix $Q$, there exists positive define symmetric matrix $P$ that satisfies Lyapunov equation $P A+A^{\mathrm{T}} P=-Q$. Therefore, there exists a quadratic Lyapunov function,

$$
V(\eta)=\eta^{\mathrm{T}} P \eta
$$

Thus, using $V(\eta)=\eta^{\mathrm{T}} P \eta$ as Lyapunov function candidate, and then its time derivative along the trajectories of the closed-loop systems (11) is,

$$
\begin{aligned}
\dot{V}(\eta) & =\frac{1}{\varepsilon} \eta^{\mathrm{T}}\left(P A+A^{\mathrm{T}} P\right) \eta+\frac{\partial V(\eta)}{\partial \eta} F(x, w) \\
& =-\frac{1}{\varepsilon} \eta^{\mathrm{T}} Q \eta+2 P_{n} \eta f_{n 1},
\end{aligned}
$$

where $P_{n}=\left[\begin{array}{llll}p_{n 1} & p_{n 2} & \cdots & p_{n, n+1}\end{array}\right]$.

Now, using the inequalities (5), (6), (8) and definition of $\eta_{i}$, we have,

$$
\left\|f_{n 1}\right\| \leq \boldsymbol{\kappa}_{f}^{\eta}(\varepsilon)\|\eta\|
$$

where $\boldsymbol{\kappa}_{f}^{\eta}(\varepsilon)$ is a positive constant, which is dependent on $\varepsilon$.

By Proposition 2 proposed by [10], we have, 


$$
\left\|P_{n}\right\|=\left\|P_{n}^{\mu}\right\| \mu \rightarrow 0 \quad \text { as } \quad \mu \rightarrow 0
$$

where $P_{n}^{\mu} \mu=P_{n}=\left[\begin{array}{llll}p_{n 1} & p_{n 2} & \cdots & p_{n, n+1}\end{array}\right]$.

Substituting (14) and (15) into (13), obtain,

$$
\dot{V}(\eta) \leq-\left(\frac{1}{\varepsilon} \lambda_{m}(Q)-2 \mu \kappa_{f}^{\eta}(\varepsilon)\left\|P_{n}^{\mu}\right\|\right)\|\eta\|^{2}
$$

It is obvious that there are $\mu^{* *}$ and $\varepsilon^{*}$ such that

$$
\frac{1}{\varepsilon} \lambda_{m}(Q)>2 \mu \kappa_{f}^{\eta}(\varepsilon)\left\|P_{n}^{\mu}\right\|
$$

holds for all $0<\mu<\mu^{* *}<\mu^{*}$ and $0<\varepsilon<\varepsilon^{*}$. Therefore, we have $\dot{V}(\eta) \leq 0$.

Using the fact that Lyapunov function $V(\eta)$ is a positive define function and its time derivative is a negative define function if the inequality (17) holds, we conclude that the closed-loop system (11) is stable. In fact, $\dot{V}(\eta)=0$ means $x=0$ and $\sigma=\sigma_{0}$. By invoking LaSalle's invariance principle, it is easy to know that the closed-loop system (11) is exponentially stable. As a result, the following theorem can be established.

Theorem 1: Under Assumptions 1, 2 and 3, if the system matrix $A$ is Hurwitz for all $\alpha_{i}, \beta_{j}$ and $0<\mu<\mu^{*}$ and the inequality,

$$
g_{m} \varepsilon^{-1} \mu^{-1} \alpha_{n+1} \varphi\left(a_{\Phi}\right)>|f(0, w)|
$$

holds, and then the equilibrium point $x=0$ and $\sigma=\sigma_{0}$ of the closed-loop system (11) is an exponentially stable for all $0<\mu<\mu^{* *}<\mu^{*}$ and $0<\varepsilon<\varepsilon^{*}$. Moreover, if all assumptions hold globally, then it is globally exponentially stable.

Discussion 1: From the demonstration above, it is obvious that: 1) as $\mu=1$, the design method degenerates into Singular perturbation one, and then the stability can be ensured by decreasing $\varepsilon$ to enhance the positive effect $\lambda_{m}(Q) ; 2$ ) as $\varepsilon=1$, the design method reduces to Equal ratio gain one, and then the asymptotically stable control can be achieved by decreasing $\mu$ to attenuate the negative influence $f_{n 1}$;3) as $\varepsilon$ and $\mu$ are all used as the control parameters, not only the positive effect $\lambda_{m}(Q)$ can be enhanced but also the negative influence $f_{n 1}$ can be attenuated, and then the perfect combination of Singular perturbation and Equal ratio gain methods is achieved. All these mean that this is a new control design technique since it not only inherits all the essences of Singular perturbation and Equal ratio gain techniques but also makes up for their shortcomings, and then the conservatism of control input can be improved by compromising the Power ratio coefficients $\varepsilon$ and $\mu$. Thus, it is not only more perfect in theory but also easier to tune a stable controller with the less conservatism in practice.

Discussion 2: From the statements above, it is easy to see that: Just there are two special coefficients $\mu$ and $\varepsilon$ in the controller (7), the closed-loop system (9) can be transformed into the singular perturbation form (11) with a canonical system matrix $A$, and then by Lyapunov method, theorem to ensure regionally as well as semi-globally asymptotic stability is established in terms of some bounded information. All of them synthesize a systematic control design method to deal with the uncertain nonlinear system. It is obvious that the key point of this design method originates in the controller gains, that is, $\alpha_{i}$ and $\beta_{j}$ are all multiplied by the Power ratio coefficients $\mu^{-1}$ and $\varepsilon^{-k}$. This is the reason why our method is called as Power Ratio Gain Technique.

Discussion 3: From the design procedure above, it is obvious that: 1) general integral controller (7), transformation from (9) to (11) and stability conditions (17) and (18) are all very simple; 2) so long as the bounded condition (14) is satisfied, the asymptotically stable control can be achieved; 3) by compromising the Power ratio coefficients $\varepsilon$ and $\mu$, the conservatism of control input can be improved. This demonstrates not only the striking feature of general integral control, that is, its robustness with respect to $f(x, w)$ and $g(x, w)$ but also Power ratio gain technique is a simple, practical and powerful tool to solve the control design problem of uncertain nonlinear system.

\section{Example and Simulation}

Consider the pendulum system [1] described by, 


$$
\ddot{\theta}=-a \sin (\theta)-b \dot{\theta}+c T
$$

where $a, b, c>0, \theta$ is the angle subtended by the rod and the vertical axis, and $T$ is the torque applied to the pendulum. View $T$ as the control input and suppose we want to regulate $\theta$ to $r$. Now, taking $x_{1}=\theta-r, \dot{x}_{2}=\dot{\theta}$, the pendulum system can be written as,

$$
\left\{\begin{array}{l}
\dot{x}_{1}=x_{2} \\
\dot{x}_{2}=-a \sin \left(x_{1}+r\right)-b x_{2}+c u
\end{array}\right.
$$

and then it can be verified that $u_{0}=a \sin (r) / c$ is the steady-state control that is needed to maintain equilibrium at the origin.

By the controller (7), we have,

$$
\left\{\begin{array}{l}
u=-\mu^{-1} \varepsilon^{-1}\left(\varepsilon^{-1} \alpha_{1} x_{1}-\alpha_{2} x_{2}-\alpha_{3} \sinh (\sigma)\right)+4 \sin \left(x_{1}\right) / 3 \\
\dot{\sigma}=\cosh ^{-1}(\sigma) \mu^{-1} \varepsilon^{-1}\left(\varepsilon^{-1} \beta_{1} x_{1}+\beta_{2} x_{2}\right)
\end{array}\right.
$$

Thus, the closed-loop system can be written as,

$$
\varepsilon \dot{\eta}=A \eta+\varepsilon F(x, w)
$$

where

$$
\begin{gathered}
\eta=\left[\begin{array}{lll}
x_{1} & x_{2} & \sinh (\sigma)-\sinh \left(\sigma_{0}\right)
\end{array}\right]^{\mathrm{T}}, \\
A=\left[\begin{array}{ccc}
0 & 1 & 0 \\
-c \mu^{-1} \alpha_{1} & -c \mu^{-1}\left(\alpha_{2}+c^{-1} \mu \varepsilon b\right) & -c \mu^{-1} \alpha_{3} \\
\mu^{-1} \beta_{1} & \mu^{-1} \beta_{2} & 0
\end{array}\right], \\
F(x, w)=\left[\begin{array}{lll}
0 & a \sin (r)-a \sin \left(x_{1}+r\right)+4 c / 3 \sin \left(x_{1}\right) & 0
\end{array}\right]^{\mathrm{T}} .
\end{gathered}
$$

The normal parameters are $a=c=10$ and $b=2$, and in the perturbed case, $b$ and $c$ are reduced to 1 and 5 , respectively, corresponding to double the mass. Thus, we have,

$$
\left\|a \sin (r)-a \sin \left(x_{1}+r\right)+4 c / 3 \sin \left(x_{1}\right)\right\| \leq 3.34 \varepsilon\|\eta\| .
$$

Now, the gains are taken as $\alpha_{1}=8, \alpha_{2}=5, \alpha_{3}=9, \beta_{1}=5$ and $\beta_{2}=3$, and then with $a=10, c=5$, $b=1$ and $\varepsilon=1$, the following inequality,

$$
c \alpha_{2} \alpha_{3} \beta_{2}+\mu\left(c \alpha_{2} \alpha_{1}+b \alpha_{3} \beta_{2}-\alpha_{3} \beta_{1}\right)+\mu \mu b \alpha_{1}>0
$$

holds for all $0<\mu<\infty$, and then the matrix $A$ is Hurwitz for all $0<\mu<\infty$. Thus, we obtain $\mu^{*}=\infty$.

Using $\alpha_{1}=8, \alpha_{2}=5, \alpha_{3}=9, \beta_{1}=5, \beta_{2}=3, c=5, b=1, \varepsilon=1.6$ and $\mu=1.5$ to solve Lyapunov equation $P A+A^{\mathrm{T}} P=-I$, obtain $\left\|P_{2}\right\| \approx 0.056$, and then solve the equation $1-6.68\left\|P_{2}\right\| \varepsilon^{2}=0$, we obtain $\varepsilon^{*}=1.62$. Thus, the asymptotical stability of the whole closed-loop system can be ensured for all $0<\varepsilon<1.62$ and $\mu \leq 1.5$. Therefore, taking $\mu=1.5$ and $\varepsilon=1.2$, the simulation is implemented under the normal and perturbed cases, respectively. Moreover, in the perturbed case, we consider an additive impulse-like disturbance $\mathrm{d}(t)$ of magnitude 60 acting on the system input between $30 \mathrm{~s}$ and $31 \mathrm{~s}$.

Figure 2 showed the simulation results under normal (solid line) and perturbed (dashed line) cases. From the simulation results and design procedure, the following observations can be made: 1 ) The optimum responses are almost identical before the additive impulse-like disturbance appears. This means that by Power ratio gain technique, we can tune a general integral controller with high control performance and good robustness. 2) It is also important to note that $\mu$ and $\varepsilon$ are taken as 1.5 and 1.2, respectively. This means that Power ratio gain technique can be used to improve the conservatism. All these demonstrates that not only general integral control can effectively deal with the uncertain nonlinearity but also Power ratio gain technique is a simple, practical and powerful tool to solve the control design problem of uncertain nonlinear system, and then makes the engineers more easily design a stable controller with the less conservatism. 


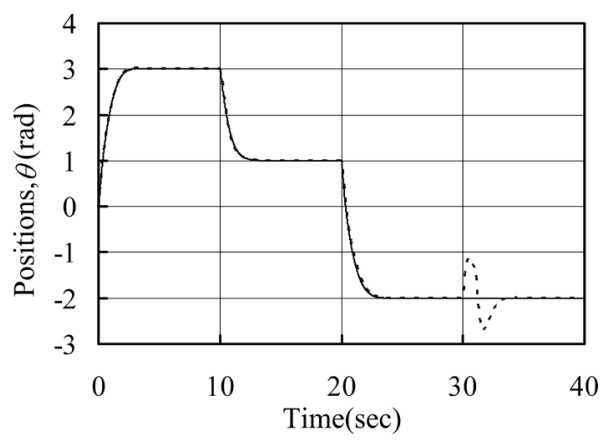

Figure 2. System output under normal (solid line) and per-turbed (dashed line) cases.

\section{Conclusion}

In conjunction with general integral control, this paper proposes a new control design technique, named Power ratio gain technique. The main contributions are: 1) the perfect combination of Singular perturbation and Equal ratio gain techniques is achieved, and then the conservatism of control input can be improved by compromising the Power ratio coefficients; 2) by Lyapunov method, theorem to ensure regionally as well as semi-globally asymptotic stability is established in terms of some bounded information. Theoretical analysis, design example and simulation results show that Power ratio gain technique is a simple, practical and powerful tool to deal with the uncertain nonlinear system. Consequently, Power ratio gain technique has not only the important theoretical significance but also the broad application prospects.

\section{References}

[1] Khalil, H.K. (2007) Nonlinear Systems. 3rd Edition, Electronics Industry Publishing, Beijing, 551, 449-453.

[2] Liu, B.S. and Tian, B.L. (2012) General Integral Control Design Based on Linear System Theory. Proceedings of the 3rd International Conference on Mechanic Automation and Control Engineering, Baotou, 5, 3174-3177.

[3] Liu, B.S. and Tian, B.L. (2012) General Integral Control Design Based on Sliding Mode Technique. Proceedings of the 3rd International Conference on Mechanic Automation and Control Engineering, Baotou, 5, 3178-3181.

[4] Liu, B.S., Li, J.H. and Luo, X.Q. (2014) General Integral Control Design via Feedback Linearization. Intelligent Control and Automation, 5, 19-23. http://dx.doi.org/10.4236/ica.2014.51003

[5] Liu, B.S., Luo, X.Q. and Li, J.H. (2014) General Integral Control Design via Singular Perturbation Technique. International Journal of Modern Nonlinear Theory and Application, 3, 173-181. http://dx.doi.org/10.4236/ijmnta.2014.34019

[6] Liu, B.S., Luo, X.Q. and Li, J.H. (2013) General Concave Integral Control. Intelligent Control and Automation, 4, 356361. http://dx.doi.org/10.4236/ica.2013.44042

[7] Liu, B.S., Luo, X.Q. and Li, J.H. (2014) General Convex Integral Control. International Journal of Automation and Computing, 11, 565-570. http://dx.doi.org/10.1007/s11633-014-0813-6

[8] Liu, B.S. (2014) Constructive General Bounded Integral Control. Intelligent Control and Automation, 5, 146-155. http://dx.doi.org/10.4236/ica.2014.53017

[9] Liu, B.S. (2014) On the Generalization of Integrator and Integral Control Action. International Journal of Modern Nonlinear Theory and Application, 3, 44-52. http://dx.doi.org/10.4236/ijmnta.2014.32007

[10] Liu, B.S. (2015) Equal Ratio Gain Technique and Its Application in Linear General Integral Control. International Journal of Modern Nonlinear Theory and Application, 4, 21-36.

[11] Liu, B.S. (2014) Nonlinear General Integral Control Design via Equal Ratio Gain Technique. International Journal of Modern Nonlinear Theory and Application, 3, 256-266.

[12] Liu, B.S., Luo, X.Q. and Li, J.H. (2014) Conventional and Added-Order Proportional Nonlinear Integral Observers. International Journal of Modern Nonlinear Theory and Application, 5, 44-52. http://dx.doi.org/10.4236/ijmnta.2014.32007 